\title{
Breaking Carbon-Fluorine Bonds with Main Group Nucleophiles
}

\author{
Greg Coates \\ Feriel Rekhroukh \\ Mark R. Crimmin*
}

Department of Chemistry, Molecular Sciences Research Hub, Imperial College London, White City, London, W12 0BZ, UK m.crimmin@imperial.ac.uk

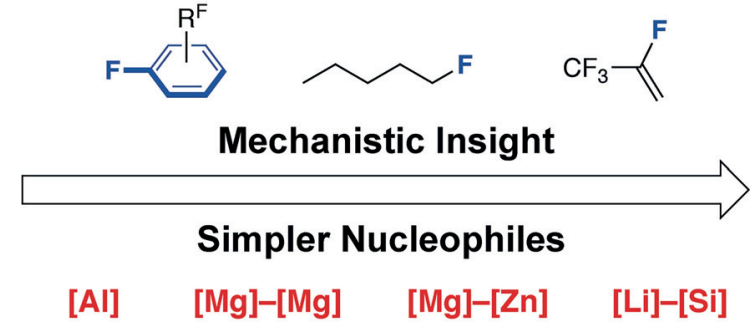

[AI]
Received: 23.09.2019

Accepted after revision: 15.10 .2019

Published online: 12.11 .2019

DOI: 10.1055/s-0039-1690738; Art ID: st-2019-a0506-a

Abstract In this Account we describe a series of new reactions that we, and others, have reported that involve the transformation of $\mathrm{C}-\mathrm{F}$ bonds into $\mathrm{C}-\mathrm{Mg}, \mathrm{C}-\mathrm{Al}, \mathrm{C}-\mathrm{Si}, \mathrm{C}-\mathrm{Fe}$ and $\mathrm{C}-\mathrm{Zn}$ bonds. We focus on the use of highly reactive main group nucleophiles and discuss aspects of reaction scope, selectivity and mechanism.

1 Introduction

1.1 The Fluorocarbon Industry and Sustainability

1.2 Production of Fluorocarbons

1.3 Properties of Fluorocarbons

1.4 Our Work

2 Results and Discussion

2.1 Low-Valent Main Group Compounds

2.1.1 Reactions with Fluoroarenes

2.1.2 Reactions with Fluoroalkanes

2.1.3 Reactions with Fluoroalkenes

2.2 Main Group Nucleophiles $\left(\mathrm{M}^{1}-\mathrm{M}^{2}\right)$

2.2.1 Reactions of $\mathrm{M}^{1}-\mathrm{M}^{2}$ Nucleophiles with Fluoroarenes

2.2.2 Reactions of $\mathrm{M}^{1}-\mathrm{M}^{2}$ Nucleophiles with Fluoroalkanes

2.2.3 Reactions of $\mathrm{M}^{1}-\mathrm{M}^{2}$ Nucleophiles with Fluoroalkenes

3 Summary and Perspective

Key words fluorine, fluorocarbons, main group, reaction mechanisms, $\mathrm{C}-\mathrm{F}$ bond activation

\section{Introduction}

\subsection{The Fluorocarbon Industry and Sustainability}

The fluorochemicals industry improves our quality of life. Fluorinated organic molecules play a pivotal role in chemical manufacture. Among their many uses they find applications as refrigerants, aerosols, in polymeric materials, as solvents, and as surfactants. For example, fluorinated polymers (Teflon) and fluorinated gases (HFCs) are commonplace in UK households, businesses and the automotive sector. ${ }^{1}$ Fluorocarbons are also found in health and farming sectors and it has been estimated that approximately $20-$ $25 \%$ of pharmaceuticals and $30-40 \%$ of agrochemicals contain at least one fluorine atom. ${ }^{2}$

Despite its clear benefits, the fluorochemicals industry is not sustainable. Nearly all fluorocarbons on our planet are synthetic. The vast majority of naturally occurring fluorine is in the form of inorganic fluoride, present in mineral forms based on abundant main group metals such as fluorspar $\left(\mathrm{CaF}_{2}\right)$ and cryolite $\left(\mathrm{Na}_{3} \mathrm{AlF}_{6}\right)$. The fluorochemical industry is now optimised to extract and process sources of inorganic fluoride to make useful organic fluorocarbons. The latter molecules and materials are often treated as single-use, being disposed of at the end of their useful life creating waste and environmental damage.

The problem of disposal is particularly acute for the refrigerants industry. While second-generation refrigerants (CFCs) created a hole in the ozone layer, third-generation refrigerants (HFCs) are potent contributors to climate change. Strict governmental legislation of these fluorinated gases has been in place for some time. The Montreal protocol has been highly effective in tackling ozone depletion and most recently this treaty has been modified (Kigali Amendment: effective January 1, 2019) to introduce regulation that seeks to reduce HFCs by $>80 \%$ by 2050 . Hydrofluoroolefins (HFOs) are now being marketed as direct replacements for HFCs as green alternatives with low globalwarming potentials. Not only is the long-term effect of HFOs, and their decomposition products such as trifluoroacetic acid, on the environment unclear but many companies are now selling mixtures of HFCs and HFOs.

There is an immediate need for sustainable approaches in the fluorochemicals industry. The recycling, repurposing and reuse of refrigerants such as HFCs and HFOs hold the potential to reduce waste, cost and environmental damage. 

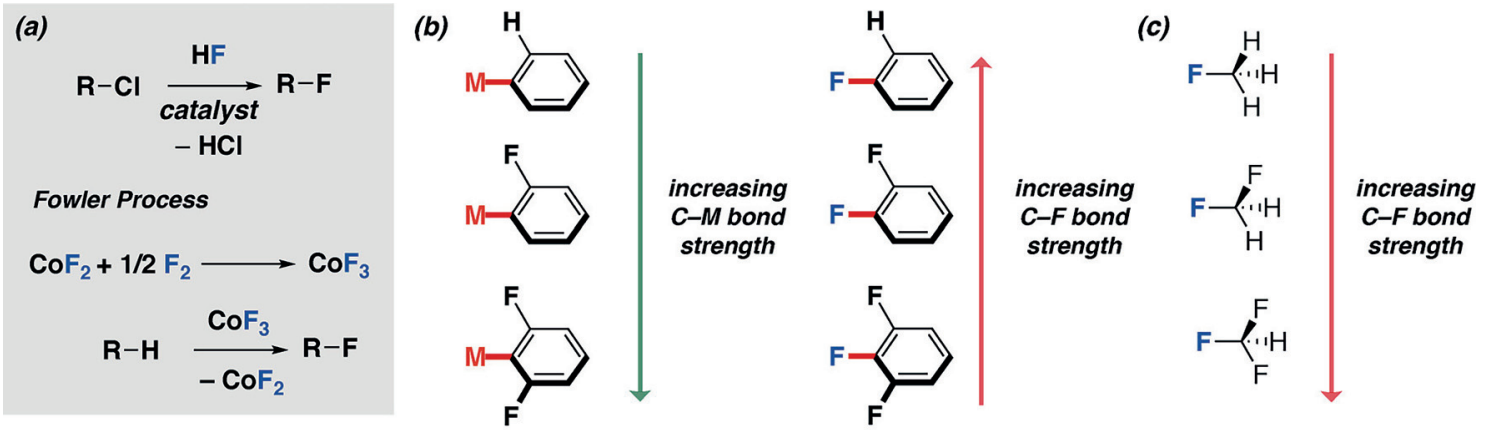

Figure 1 (a) Production of fluorocarbons from chlorocarbons and hydrocarbons. (b) Trends in C-M and C-F bond strengths in fluorobenzenes. (c) Trends in $\mathrm{C}-\mathrm{F}$ bond strengths in fluoroalkanes.

The key challenge here is the development and implementation of new chemical methods that use the C-F bond as a functional group.

\subsection{Production of Fluorocarbons}

On industrial scales, nearly all fluorocarbons are prepared from HF, which is in turn obtained in an aqueous form through reaction of fluorite $\left(\mathrm{CaF}_{2}\right)$ with sulfuric acid $\left(\mathrm{H}_{2} \mathrm{SO}_{4}\right)$. $\mathrm{HF}$ can be used as a source of $\mathrm{F}^{-}$and in the presence of a metal catalyst (often chromium-based) can react with chlorocarbons to form fluorocarbons or chlorofluorocarbons (Figure 1a).

The main routes to selective fluorination in the life-sciences sector are via the Swarts halogen-exchange process and the Balz-Schiemann process. These focus on fluoroaromatics and trifluoromethyl aromatics, respectively, and both use anhydrous HF as the fluorinating agent. ${ }^{3}$ Alternatively, electrolysis of HF yields $F_{2}$, which although a poor fluorinating reagent by itself due to its extreme oxidising nature, can be combined with $\mathrm{CoF}_{2}$ to effect the fluorination of hydrocarbons in the Fowler process. ${ }^{4}$ The Fowler process has two main steps, the reaction of $\mathrm{CoF}_{2}$ with $\mathrm{F}_{2}$ to generate cobalt trifluoride $\left(\mathrm{CoF}_{3}\right)$, which is then heated with a hydrocarbon substrate to perform the fluorination (Figure 1a). These conditions lead to multiple fluorination events and yield organic compounds with high fluorine content, typically perfluorinated or polyfluorinated molecules. The $\mathrm{CoF}_{2}$ by-product can be recycled. Anhydrous hydrofluoric acid can also be used as a fluorinating agent in an electrochemical reaction, called the Simmons process, to generate aliphatic $\mathrm{C}-\mathrm{F}$ bonds from $\mathrm{C}-\mathrm{H}$ bonds. ${ }^{5}$

\subsection{Properties of Fluorocarbons}

The properties of fluorocarbons can be traced to unique aspects of the element fluorine and its position on the periodic table. Fluorine has a large first ionisation energy ( $I E_{1}=$ $\left.401.2 \mathrm{kcal} \mathrm{mol}^{-1}\right)$ and electron affinity $\left(E_{\mathrm{EA}}=78.3 \mathrm{kcal} \mathrm{mol}^{-}\right.$ $\left.{ }^{1}\right)$ and is the most electronegative element known $\left(X^{p}=\right.$ 4.0). ${ }^{6}$ The fluorine atom has one of the smallest radii $\left(r_{\mathrm{vdW}}=\right.$ $1.47 \AA)$, second only to the hydrogen atom $\left(r_{v d w}=1.20 \AA\right)$. Hence, it is suitable to consider substitution of $\mathrm{H}$ for $\mathrm{F}$ in hydrocarbons. Due to the large electronegativity difference between carbon and fluorine $\left(\Delta X^{p}=1.5\right)$ however, $C-F$ bonds are significantly different from $\mathrm{C}-\mathrm{H}$ bonds. $\mathrm{C}-\mathrm{F}$ bonds are polar and exceptionally strong. In fact, this is the strongest single bond between carbon and any element. For example, the $\mathrm{C}-\mathrm{F}$ bond-dissociation energy of fluorobenzene $\left(\mathrm{C}_{6} \mathrm{H}_{5} \mathrm{~F}\right)$, fluoroethene $\left(\mathrm{CHF}=\mathrm{CH}_{2}\right)$ and fluoromethane $\left(\mathrm{CH}_{3} \mathrm{~F}\right)$ are $127.2 \pm 0.7,123.3 \pm 0.8$ and $115 \pm 4 \mathrm{kcal} \mathrm{mol}^{-1}$ respectively. ${ }^{7}$ These are all larger than the $\mathrm{C}-\mathrm{H}$ bond-disso-

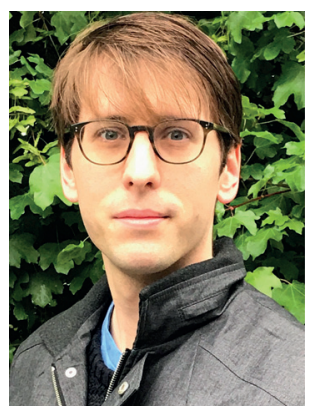

Mark R. Crimmin graduated from Imperial College London in 2004 and completed an MSc by research in organic synthesis at Bristol University under the supervision of Prof. V. K. Aggarwal. He received his $\mathrm{PhD}$ in main group chemistry and catalysis from Imperial College London in 2008 supervised by Prof.
Michael Hill (now at University of Bath) and Prof. Tony Barrett. In the same year, he was awarded a Royal Commission for the Exhibition of 1851 research fellowship which he took to University of California, Berkeley (USA) to study with Prof. Bob Bergman and Prof. Dean Toste. In 2011, he returned to London as a Royal Society University Research Fellow, initially at University College London and now back at Imperial. He was appointed as a lecturer in 2011 , senior lecturer in 2016 and reader (associate professor) in 2019. 
ciation energy in the corresponding hydrocarbons benzene, ethene and methane of $112.9 \pm 0.6,110.7 \pm 0.7$ and $104.9 \pm 0.1 \mathrm{kcal} \mathrm{mol}^{-1}$ respectively. ${ }^{7}$

Incorporation of fluorine atoms into organic compounds influences the properties of these molecules as a whole. For example, substitution of $\mathrm{H}$ for $\mathrm{F}$ in benzene results in a strengthening of the $\mathrm{C}-\mathrm{H}$ bonds adjacent to the newly installed $\mathrm{C}-\mathrm{F}$ bond. $\mathrm{C}-\mathrm{H}$ bond-dissociation energies in fluorobenzenes increase with increasing ortho fluorine substitution. A similar trend, but with a stronger correlation, is observed for $\mathrm{C}-\mathrm{M}$ bonds (Figure $1 \mathrm{~b}){ }^{8-10}$ In contrast, the opposite trend is observed for C-F bond strengths. Macgregor and Whittlesey have examined this effect for a number of polyfluorinated benzenes. ${ }^{11}$ The $\mathrm{C}-\mathrm{F}$ bond strengths in fluorobenzenes have been calculated to decrease by $\sim 1.8,0.5$ and $0.2 \mathrm{kcal} \mathrm{mol}^{-1}$ upon substitution of $\mathrm{H}$ atoms by $\mathrm{F}$ atoms in the ortho-, meta- and para-position respectively (Figure 1c). In combination, these trends lead to substrate bias in CF activation of fluoroarenes due to a thermodynamic orthofluorine effect. In fluorobenzenes, the weakest C-F bonds are those flanked by additional ortho fluorine atoms, and cleavage of these bonds to form organometallics results in the formation of products with the strongest $\mathrm{C}-\mathrm{M}$ bonds.

Similar substrate bias is important for fluoroalkanes too. The $\mathrm{C}-\mathrm{F}$ bond strengths in fluoromethanes follow the trend $\mathrm{CF}_{3} \mathrm{H}>\mathrm{CF}_{2} \mathrm{H}_{2}>\mathrm{CFH}_{3}$. Increasing fluorine content results in a mutually re-enforcing effect with the polarisation of each $\mathrm{C}-\mathrm{F}$ bond resulting in increased partial positive charge on carbon and increase in the ionic character and bond-dissociation energy of each of the C-F bonds (Figure 1c). ${ }^{6}$ As a result, $\mathrm{CF}_{3}$ and $\mathrm{CF}_{2} \mathrm{H}$ groups are some of the most chemically inert functional groups known in chemistry and it is not surprising they have found widespread application in industry-applied compounds such as hydrofluorocarbon (HFC) refrigerants and active pharmaceutical ingredients (APIs).

Despite its extreme electronegativity, the strong C-F bond makes fluorine a poor leaving group. Rates of substitution of $\mathrm{S}_{\mathrm{N}} 2$ reactions of haloalkanes, $\mathrm{R}-\mathrm{X}$, increase with the trend $\mathrm{X}=\mathrm{I}>\mathrm{Br}>\mathrm{Cl}>\mathrm{F} .{ }^{6}$ Nevertheless, fluorocarbons can react with suitable nucleophiles and bases. Mechanistic pathways involving closed-shell species that have been observed include, but are not limited to (Figure 2):

- $\mathrm{CS}_{\mathrm{N}} \mathrm{Ar}, \mathrm{S}_{\mathrm{N}} \mathrm{Ar}$, benzyne formation (fluoroarenes)

- $\mathrm{S}_{\mathrm{N}} 2$, fluoride abstraction, $\mathrm{E}_{1} \mathrm{CB}$ (fluoroalkanes)

$-\mathrm{S}_{\mathrm{N}} \mathrm{V}, \mathrm{S}_{\mathrm{N}} 2^{\prime}$, addition-elimination (fluoroalkenes)

For example, $\mathrm{S}_{\mathrm{N}} \mathrm{Ar}$ has been used widely in synthesis with fluoroarenes being applied as substrates in both carbon-carbon and carbon-heteroatom bond-forming reactions. ${ }^{12,13}$ There is increasing realisation that the concerted variant of this pathway $\mathrm{CS}_{\mathrm{N}} \mathrm{Ar}$ may be more prevalent than its stepwise counterpart $S_{N} A r .{ }^{14,15}$ A number of electrontransfer mechanisms are also potentially in operation in reactions of fluorocarbons and can become accessible due to the low-lying $\sigma^{*} \mathrm{C}-\mathrm{F}$ orbital. ${ }^{16,17}$
Non-covalent interactions play an important role in determining the reactivity of fluorocarbons. These influence the structure of the ground state and can lead to the stabilisation of transition states. For example, the reactivity of fluorocarbons can be influenced by: (i) dipole-dipole interactions, (ii) dipole-charge interactions, (iii) hyperconjugation effects involving donation of electron-density into the lowlying $\sigma^{*}$ orbital of the $\mathrm{C}-\mathrm{F}$ bond, (iv) weak $\mathrm{C}-\mathrm{F} \cdots \mathrm{H}-\mathrm{X}$ hydrogen bonding interactions, and $(v)$ strong electrostatic CF...M interactions. ${ }^{6}$

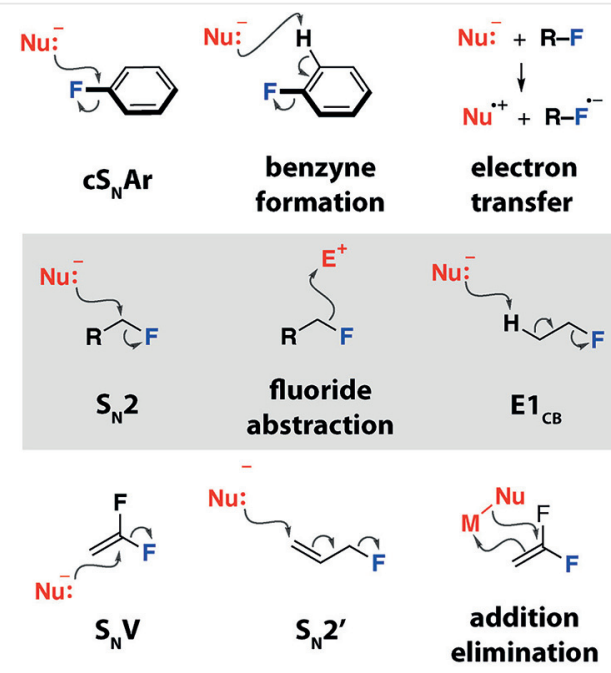

Figure 2 General reactions of fluorocarbons with nucleophiles

\subsection{Our Work}

Despite their exceptionally high bond-dissociation energies, even the most chemically robust fluorocarbons will react with suitably high-energy molecules and intermediates. We have focused on developing new reactions that transform environmentally persistent fluorocarbons into reactive chemical building blocks. Highly selective methods have been developed that transform $\mathrm{C}-\mathrm{F}$ bonds into $\mathrm{C}-\mathrm{Mg}$, C-Al, C-Si, C-Fe and C-Zn bonds. ${ }^{18,19}$ These elements are all electropositive and form extremely strong bonds with fluorine. As a result, reactions with fluorocarbons are often exergonic with reactivity patterns determined by the thermodynamic factors described above. Compounds based on these electropositive elements can form strong M...F electrostatic interactions, engaging and orientating the fluorocarbon in a suitable geometry for nucleophilic attack and hence lowering transition-state energies.

In this Account, we document part of these studies, those which use nucleophilic main group reagents and do not rely on the use of transition-metal complexes. ${ }^{20-23}$ Where possible we have highlighted the elegant research of other groups in the field that are of direct relevance to the discussion. We have omitted work on hydrodefluorination, that is the transformation of C-F to C-H bonds. ${ }^{24,25}$ We em- 
phasise some of the insight gained through development of mechanistic understanding and conclude by highlighting the challenges and opportunities that remain in this field.

\section{Results and Discussion}

\subsection{Low-Valent Main Group Compounds}

Low-valent main group reagents have proven remarkably reactive towards fluorocarbons. These compounds contain main group metals in low oxidation states and possess a small HOMO-LUMO gap. Their frontier molecular orbitals are of suitable energy and symmetry to engage in interactions with organic substrates. ${ }^{26}$ For example, the aluminium complex $\mathbf{1}$ has a structure that is analogous to an $\mathrm{N}$-heterocyclic carbene and possesses an aluminium-based lone pair and an orthogonal vacant 3p-orbital (Figure 3a). ${ }^{27}$ The poor $2 \mathrm{p} / 3 \mathrm{p}$ overlap between the nitrogen lone pairs of the ligand and aluminium-based orbitals, in combination with the low electronegativity of aluminium, make this complex both a good nucleophile and a good Lewis acid. Its mode of reactivity combines these two facets and $\mathbf{1}$ can be described as a transition-metal mimic.

\subsubsection{Reactions with Fluoroarenes}

In 2015, we reported that the aluminium(I) complex 1 reacts with fluoroarenes under mild conditions to form organoaluminium compounds (Figure $3 \mathrm{~b}){ }^{28}$ The reaction results in the breaking of a $\mathrm{C}-\mathrm{F}$ bond with formation of new $\mathrm{Al}-\mathrm{C}$ and $\mathrm{Al}-\mathrm{F}$ bonds on the same aluminium centre and is considered as an oxidative addition of the fluorocarbon to Al. Our study was initiated based on an observation that the group had reported in 2012 . We showed that closely related organoaluminium compounds were formed as side-products in the zirconium-catalysed reaction of fluorocarbons with the aluminium(III) dihydride $\mathbf{2 b}$ (Figure 3a). ${ }^{29,30}$ We have since reported an efficient Pd-based catalyst system for selective $\mathrm{C}-\mathrm{F}$ (and $\mathrm{C}-\mathrm{H}$ ) to $\mathrm{C}-\mathrm{Al}$ bond transformations (a)

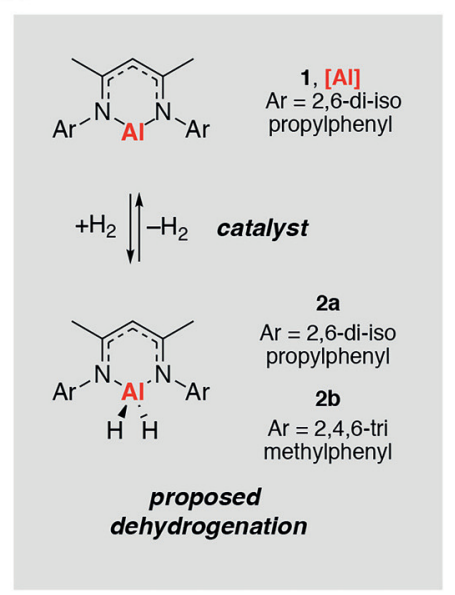

(b)

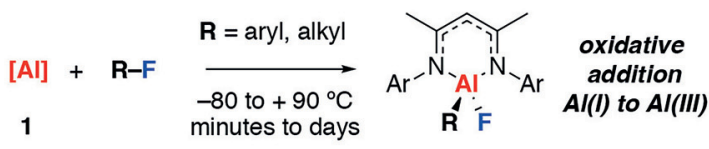

substrate scope: fluoroarenes

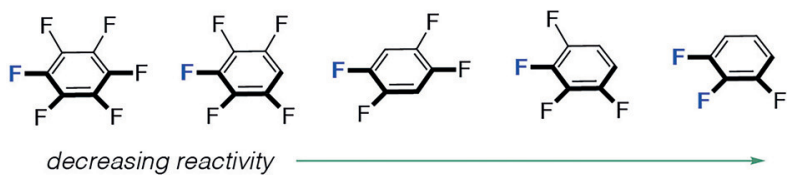

substrate scope: fluoroalkanes

(c) $\quad$ rate $=k[1][\mathbf{R}-\mathrm{F}]$ $\Delta \mathrm{H}^{\ddagger}=+13.6(1) \mathrm{kcal} \mathrm{mol}^{-1}$ $\Delta S^{\ddagger}=-27(1) \mathrm{cal} \mathrm{K}^{-1} \mathrm{~mol}^{-1}$ $\Delta \mathrm{G}^{\ddagger}=+21.8(1) \mathrm{kcal} \mathrm{mol}^{-1}$ (d)<smiles>Fc1cc(F)c(F)cc1F</smiles>

oxidative addition ( $\mathrm{CS}_{N}$ Ar-like mechanism)

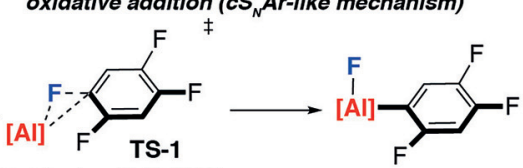

$\Delta \mathrm{G}^{\ddagger}=+28.3 \mathrm{kcal} \mathrm{mol}^{-1}(\mathrm{~B} 3 \mathrm{LYP})$

$\Delta \mathrm{G}^{\ddagger}=+24.5 \mathrm{kcal} \mathrm{mol}^{-1}$ (B3LYP-D3, model ligand)

$\Delta \mathrm{G}^{\ddagger}=+23.0 \mathrm{kcal} \mathrm{mol}^{-1}$ (B3PW91-GD3)

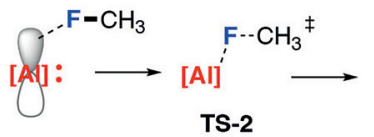

$\Delta \mathrm{G}^{\ddagger}=+26.5 \mathrm{kcal} \mathrm{mol}^{-1}$ (B3LYP-D3, model ligand)

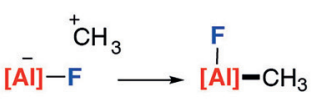

stepwise fluoride abstraction

step (e)

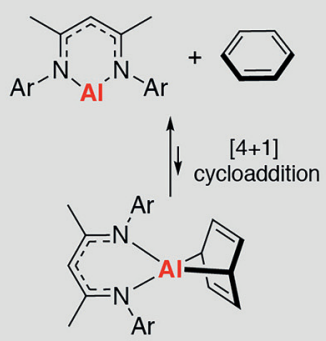

(proposed equilibrium based on DFT calculations)

Figure 3 (a) Structure of low-valent aluminium reagent 1 along with related aluminium dihydride $\mathbf{2}$. (b) Reaction of $\mathbf{1}$ with fluoroarenes and fluoroalkanes. (c) Experimentally determined activation parameters for the reaction of 1 with 1,2,3,4-tetrafluorobenzene. ${ }^{35}$ (d) Proposed mechanisms for reaction of 1 with fluoroarenes and fluoroalkanes based on DFT studies. ${ }^{41,42}$ (e) Proposed equilibrium between 1 and a [4+1] cycloaddition product based on DFT calculations. ${ }^{39}$ 
with $\mathbf{2 b}{ }^{31}$ We hypothesised that low-valent aluminium(I) complexes may be intermediates in these reactions and capable of effecting $\mathrm{C}-\mathrm{F}$ bond activation in the absence of the transition metal. Related oxidative addition reactions of $\mathrm{H}-$ $\mathrm{H}, \mathrm{H}-\mathrm{Si}$, acidic $\mathrm{C}-\mathrm{H}, \mathrm{B}-\mathrm{H}$ and $\mathrm{Al}-\mathrm{H}$ bonds to $\mathbf{1}$ were reported by Nikonov and co-workers in $2014 .^{32}$ Furthermore, isostructural $\mathrm{Si}(\mathrm{II})$ and $\mathrm{Ge}$ (II) reagents had been shown to undergo oxidative addition reactions with fluoroarenes. ${ }^{33,34}$

Subsequent to our report, Nikonov and co-workers documented the reactions of $\mathbf{1}$ with a number of fluoroarenes. ${ }^{35}$ The combined reaction scope from the two studies includes fluorinated benzenes with 3-6 fluorine atoms (Figure 3b). ${ }^{28,35}$ Reaction times and temperatures decrease with increasing fluorine content, consistent with the most electron-deficient fluoroarenes being the most reactive toward 1. For example, $\mathrm{C}_{6} \mathrm{~F}_{6}$ reacts with 1 within 15 minutes at $25{ }^{\circ} \mathrm{C}$, while $1,2,3-\mathrm{C}_{6} \mathrm{~F}_{3} \mathrm{H}_{3}$ requires heating for 96 hours at $80{ }^{\circ} \mathrm{C}$ to reach reasonable conversions. The regioselectivity of the oxidative addition step is consistent with the thermodynamic arguments set out in the introduction (section 1.3). Reactive $\mathrm{C}-\mathrm{F}$ bonds are those flanked by one or more fluorine atom, and as such these are not only the weakest $\mathrm{C}-\mathrm{F}$ bonds but also those which lead to the formation of the strongest $\mathrm{C}-\mathrm{Al}$ bonds. These reactive sites are also expected to lead to the most stable transition states, as the partial positive charge that builds up on the reactive carbon centre will be accommodated by the inductive effect of adjacent $F$ atoms.

Nikonov and co-workers investigated the kinetics of the addition of $\mathbf{1}$ to $1,2,3,4-\mathrm{C}_{6} \mathrm{~F}_{4} \mathrm{H}_{2}$ under pseudo-first-order conditions (excess substrate) at $295.1 \mathrm{~K}$ and demonstrated that the reaction is first-order in [1]. Further variation of initial concentrations of the fluorocarbon suggests the reaction is also first-order in substrate. An Eyring analysis across 264.1 to $303.1 \mathrm{~K}$ temperature range allowed activation parameters of $\Delta \mathrm{H}^{\ddagger}=+13.6 \pm 1 \mathrm{kcal} \mathrm{mol}^{-1}$ and $\Delta \mathrm{S}^{\ddagger}=-27 \pm 1 \mathrm{cal} \mathrm{K}^{-1}$ $\mathrm{mol}^{-1}$, the corresponding $\Delta \mathrm{G}^{\ddagger}(298 \mathrm{~K})=21.8 \pm 1 \mathrm{kcal} \mathrm{mol}^{-1} \cdot 35$ The data are consistent with a rate-limiting step in which both 1 and fluoroarene combine in a highly ordered transition state and have been interpreted as evidence for a concerted oxidative addition mechanism (Figure 3c).

A number of mechanisms have been proposed for the reaction of 1 with simple organic substrates (Figure 3d). For example, the reaction of $\mathbf{1}$ with $\mathrm{H}_{2}$ has been calculated to occur by a concerted pathway. This has been described in detail as an asynchronous concerted process. ${ }^{36}$ The potential energy surface involves dihydrogen approaching the vacant 3p orbital on $\mathrm{Al}$, an event that leads to the polarisation of the $\mathrm{H}^{\delta+} \ldots \mathrm{H}^{\delta-}$ bond which can then undergo nucleophilic attack by the $\mathrm{Al}$ carbenoid followed by migration of the remaining hydride to Al. Overall the process is an oxidative addition. There is no computational support for a stable dihydrogen complex as an intermediate and, while asynchronous, the reaction proceeds by a single transition state. On modification of the substrate to $\mathrm{X}-\mathrm{H}$ bonds ( $\mathrm{X}=\mathrm{C}, \mathrm{Si}, \mathrm{Ge}, \mathrm{Sn}$;
$\mathrm{O}, \mathrm{S}, \mathrm{N}, \mathrm{P}$ ) two concerted transition states are possible. The favoured one involves the $\mathrm{X}$ atom in the axial position, approaching the vacant orbital of $\mathrm{Al}^{37}$ While some have proposed that reactivity trends $\left(\Delta \mathrm{E}^{\ddagger}: \mathrm{C}>\mathrm{Si}>\mathrm{N}>\mathrm{P} ; \mathrm{O}>\mathrm{Si}\right)$ are determined by the $\mathrm{H}-\mathrm{X}$ bond strengths, analysis with the activation-strain model suggests a more complex explanation. ${ }^{38}$ Lower-energy transition states are those in which the interaction energy is minimised due to increased orbital and electrostatic interactions. Both factors can be correlated with the energy of the $\sigma^{*}$ orbital of the $\mathrm{X}-\mathrm{H}$ bond. Lower-energy $\sigma^{*}(\mathrm{X}-\mathrm{H})$ orbitals lead to lower-energy transition states. Vanka and Jain have proposed an alternative unusual mechanism for $\mathrm{X}-\mathrm{H}$ bond activation with 1. ${ }^{39}$ Based on the reactions being reported in benzene as a solvent, they suggest a stepwise process involving benzene itself. The 1,4-cycloaddition of $\mathbf{1}$ with benzene forms an unstable [4+1] intermediate (Figure 3e) which in turn is capable of $\mathrm{X}-\mathrm{H}$ bond activation by addition across one of the newly formed $\mathrm{Al}-\mathrm{C}$ bonds. Subsequent hydride elimination and rearomatisation re-forms benzene and yields the $\mathrm{H}-\mathrm{X}$ addition product. While the high-energy [4+1] cycloaddition product has now been trapped by coordination to a second metal, ${ }^{40}$ it is highly unlikely that this pathway operates for the fluorocarbons described herein, as reversible $\mathrm{C}-\mathrm{C}$ and $\mathrm{C}-\mathrm{F}$ bond formation would be required as part of the mechanism.

Two separate computational studies have concluded that fluoroarenes react with $\mathbf{1}$ by a concerted pathway (Figure $3 \mathrm{~d}) .{ }^{41,42}$ The calculated TS-1 energy for reaction of $\mathbf{1}$ with $1,2,4,5-\mathrm{C}_{6} \mathrm{~F}_{4} \mathrm{H}_{2}$ is $\Delta \mathrm{G}^{\ddagger}(298 \mathrm{~K})=28.3 \mathrm{kcal} \mathrm{mol}^{-1}$ using the B3LYP functional. TS-1 involves approach of the fluorine atom to the axial position of the aluminium complex and evolves from an unstable encounter complex involving a weak long-distance interaction between $\mathrm{Al}$ and $\mathrm{F}(\sim 4 \AA) .{ }^{41}$ While using a simplified ligand system and modifying the functional to consider dispersion effects B3LYP-D3 yields a lower activation barrier of $\Delta \mathrm{G}^{\ddagger}(298 \mathrm{~K})=24.5 \mathrm{~mol}^{-1}$, this still remains higher than the experimentally determined value for 1,2,3,4-tetrafluorobenzene. ${ }^{42}$ Using this latter level of theory, it has been suggested that a stepwise mechanism involving fluoride abstraction from the arene and recombination of the charged fragments is plausible but less favourable than the concerted pathway. We recalculated TS-1 using alternative computational functionals including B3PW91-GD3 and found a similar activation barrier of $\Delta \mathrm{G}^{\ddagger}$ $(298 \mathrm{~K})=23.0 \mathrm{kcal} \mathrm{mol}^{-1}$.

\subsubsection{Reactions with Fluoroalkanes}

The activation and functionalisation of $\mathrm{sp}^{3} \mathrm{C}-\mathrm{F}$ bonds of fluoroalkanes represents an important and largely unsolved challenge. While there are abundant examples of oxidative addition processes for fluoroarenes, the addition of $\mathrm{sp}^{3} \mathrm{C}-\mathrm{F}$ bonds to transition metals is problematic. The lack of charge stabilisation in the transition state for $\mathrm{sp}^{3} \mathrm{C}-\mathrm{F}$ bond 
breaking means that defined oxidative addition reactions are scarce. Furthermore, the resulting metal-alkyl bonds can be unstable with respect to $\beta$-hydride elimination abetted through the availability of metal $d$ orbitals and agostic interactions. Nevertheless, transition-metal-mediated $\mathrm{sp}^{3} \mathrm{C}-\mathrm{F}$ activation is not completely without precedent. For example, in 2011 fluoromethane was shown to undergo a formal oxidative addition to an iridium pincer complex. ${ }^{43} \mathrm{~A}$ direct oxidative $\mathrm{C}-\mathrm{F}$ process was found to be high in energy and shown to be a disfavoured pathway. Instead, oxidative addition involves a more complex stepwise mechanism and initial oxidative addition of a $\mathrm{C}-\mathrm{H}$ bond to iridium.

Despite the challenging nature of the transformation, 1 undergoes facile oxidative addition reactions with $1^{\circ}$ and $2^{\circ}$ fluoroalkanes. ${ }^{28,35}$ Substrates include 1-fluorohexane, 1-fluoropentane and fluorocyclohexane. Reactions proceed within 15 minutes at $25^{\circ} \mathrm{C}$ and lead to the formation of new aluminium alkyl complexes. The latter are characterised by diagnostic high-field resonances in the ${ }^{1} \mathrm{H}$ NMR spectrum evident of the methylene or methine proton environments adjacent to the electropositive aluminium centre. The reaction scope has yet to be extended beyond a handful of substrates or to $3^{\circ}$ fluoroalkanes.
While a detailed mechanistic study has not been conducted, both stepwise and concerted mechanisms have been proposed based on DFT calculations. Hwang and coworkers modelled the concerted oxidative addition pathway. ${ }^{41}$ Wang and Pitsch considered both concerted oxidative addition and the fluoride abstraction pathway and concluded that the latter stepwise pathway should be favoured based on the low energy of TS-2 (Figure 3d). ${ }^{42}$ Experimental support for a long-lived carbocation is limited.

\subsubsection{Reactions with Fluoroalkenes}

In 2018, our group expanded the scope of reactivity of $\mathbf{1}$ to fluoroalkenes. ${ }^{44}$ The reactions included both perfluorinated substrates such as hexafluoropropene (HFP) and also polyfluorinated molecules including the industrially relevant hydrofluoroolefins HFO-1234yf and HFO-1234ze (Figure $4 a$ ). These tetrafluoroalkenes are being manufactured and promoted as next-generation refrigerants. The inclusion of both $\mathrm{sp}^{2}$-hybridised and $\mathrm{sp}^{3}$-hybridised $\mathrm{C}-\mathrm{F}$ bonds within these substrates affords an opportunity to study the issues of chemoselectivity and regioselectivity.

(a)

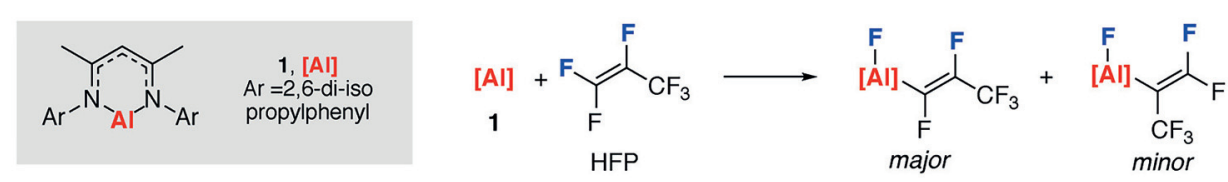

[AI]<smiles>C=C(F)C(F)(F)[Al]C(F)(F)C(F)(F)F</smiles>

HFO-1234yf

$s p^{3} C-F$ bond activation (alkene transposition)

[AI]

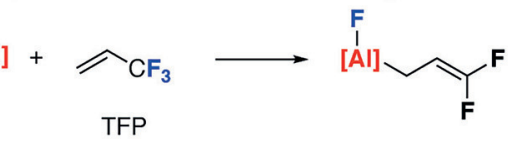

stereochemical labelling experiments

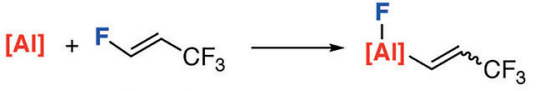
E-HFO-1234ze $\quad 4: 1 E: Z$

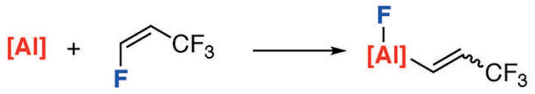

$$
\text { Z-HFO-1234ze } \quad 0.9: 1 \mathrm{E:Z}
$$

(b) oxidative addition ( $S_{N}$-like mechanism)<smiles>FC(F)(F)/C=C/CC1CCCCC1</smiles>

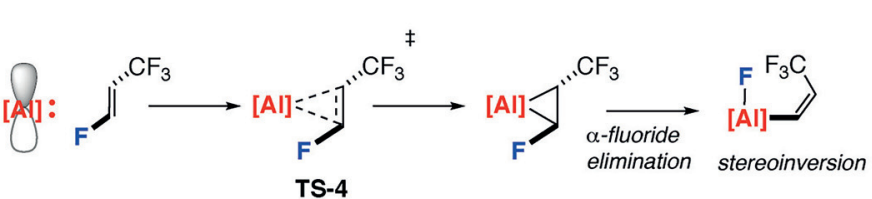

(c)

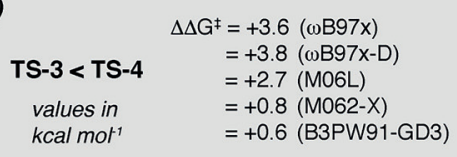

(d)

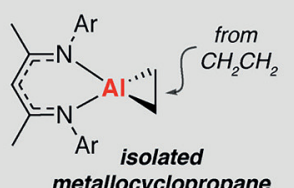

Figure 4 (a) Reactions of 1 with fluoroalkenes including industrially relevant HFOs. (b) Proposed mechanisms for C-F activation based on DFT studies. (c) Comparison of the energies of TS-3 and TS-4 based on a series of computational methods. (d) An isolated metallocyclopropane of relevance to the mechanistic discussion. 
In all cases, oxidative addition is selective for $\mathrm{sp}^{2} \mathrm{C}-\mathrm{F}$ bonds over $\mathrm{sp}^{3} \mathrm{C}-\mathrm{F}$ bonds. Only the complete exclusion of the former reactive sites from the substrate leads to reactions involving $\mathrm{CF}_{3}$ groups. For example, 1 reacts with HFP at two different $\mathrm{sp}^{2} \mathrm{C}-\mathrm{F}$ sites with conservation of the $\mathrm{CF}_{3}$ group; the major product is derived from reaction of the terminal $\mathrm{sp}^{2} \mathrm{C}-\mathrm{F}$ bond trans to the $\mathrm{CF}_{3}$ group, whereas the minor product is derived from reaction of the internal $\mathrm{sp}^{2} \mathrm{C}-\mathrm{F}$ bond. HFO-1234yf reacts exclusively at the internal $\mathrm{sp}^{2} \mathrm{C}-\mathrm{F}$ bond, while 3,3,3-trifluoroprop-1-ene undergoes allylic $\mathrm{sp}^{3} \mathrm{C}-\mathrm{F}$ bond activation leading to formation of a gemdifluorovinyl group due to transposition of the $\mathrm{C}=\mathrm{C}$ double bond. HFO-1336-mzz does not react with 1 to form an organometallic intermediate, but rather undergoes a double C-F activation to form an aluminium difluoride and the $s$ isomer of 1,1,4,4-tetrafluorobuta-1,3-diene (Figure 5).

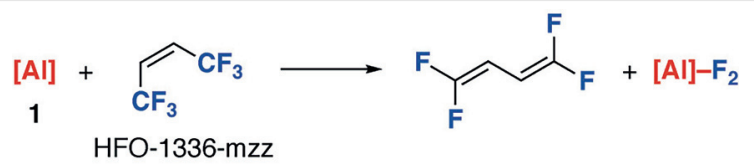

Figure 5 Reaction of 1 with HFO-1336-mzz to form a fluorinated butadiene

Reactions of pure samples of E-HFO-1234ze and Z-HFO$1234 z e$ with 1 provide further insight as the stereochemistry about the $\mathrm{C}=\mathrm{C}$ bond in the product yields information about the mechanistic pathway (Figure 4a). In both cases, the major product evolves from a pathway involving stereoretention of the alkene geometry. Nevertheless, the reactions are not stereospecific and occur with a degree of erosion of the stereochemistry. The degree of stereoerosion is greater for reaction of Z-HFO-1234ze than E-HFO-1234ze. Monitoring these reactions by ${ }^{19} \mathrm{~F}$ NMR spectroscopy reveals that the $E: Z$ ratios of the products do not change as a function of time; furthermore, attempts to equilibrate pure samples of the $E$-isomer of the product to the $Z$-isomer with heat or light were ineffective.

There are a number of interesting features of this study that are complementary to the mechanistic aspects of the reaction of 1 with fluoroarenes and fluoroalkanes. These include the partial erosion of $E / Z$ stereochemistry, the activation of both $\mathrm{sp}^{2}$-hybridised and $\mathrm{sp}^{3}$-hybridised $\mathrm{C}-\mathrm{F}$ bonds and, in certain cases, the transposition of the $C=C$ double bond. The data are consistent with more than one mechanism operating. DFT calculations were used to interrogate the potential pathways for C-F activation (Figure $4 \mathrm{~b}$ ). A concerted pathway involving a stereoretentive oxidative addition of the C-F bond by TS-3 was identified alongside stepwise processes involving alkene coordination to $\mathbf{1}$. Alkene coordination to $\mathbf{1}$ forms a metallocyclopropane intermediate which can act as a precursor to $\mathrm{C}-\mathrm{F}$ bond activation by either $\alpha$-fluoride or $\beta$-fluoride elimination. The former leads to $\mathrm{sp}^{2} \mathrm{C}-\mathrm{F}$ cleavage and inversion of the alkene stereochemistry, while the latter leads to $\mathrm{sp}^{3} \mathrm{C}-\mathrm{F}$ cleavage and transposition of the $\mathrm{C}=\mathrm{C}$ bond. For the stepwise processes, these calculations suggest that metallocyclopropane formation by TS-4 is rate-limiting with facile C-F activation occurring after coordination to $\mathbf{1}$.

Comparison of these pathways for the reaction of $\mathbf{1}$ with E-HFO-1234ze using a number of DFT functionals ( $\omega$ B97x, $\omega B 97 x D, M 062 x, M 06 L$, and B3PW91) revealed that TS-3 and TS-4 transition states for the concerted stereoretentive mechanism and stepwise stereoinversion mechanisms respectively are close enough $\left(\Delta \Delta \mathrm{G}^{\ddagger}=0.6-3.8 \mathrm{kcal} \mathrm{mol}^{-1}\right)$ in energy to suggest that both may be operating (Figure $4 \mathrm{c}){ }^{44}$ Further support for the proposed pathway involving alkene coordination was gained from reaction of $\mathbf{1}$ with a series of alkenes and isolation of the corresponding metallocyclopropane complexes (Figure 4d). ${ }^{45}$ Hence, formation of metallocyclopropane intermediates in the reaction of $\mathbf{1}$ with fluoroalkenes is a viable proposal.

\subsection{Main Group Nucleophiles $\left(\mathrm{M}^{1}-\mathrm{M}^{2}\right)$}

In parallel to investigating the reactions of fluorocarbons with single-site aluminium compounds, we have been studying their reactions with reagents that contain metalmetal or metal-semi-metal bonds. These reactions proceed by a 1,2-addition of the $\mathrm{C}-\mathrm{F}$ bond across the $\mathrm{M}^{1}-\mathrm{M}^{2}$ bond with the most electropositive metal acting as a fluoride acceptor and the least electrophilic metal as the nucleophilic site. The reactions result in the cleavage of the metal-metal bond and in most cases two products, a metal fluoride and an organometallic. In the case of $\mathrm{M}^{1}=\mathrm{M}^{2}$ the reaction can formally be assigned as an oxidative addition of the fluorocarbon to the main group reagent. Nevertheless, both these cases and $\mathrm{M}^{1} \neq \mathrm{M}^{2}$ both show the trademarks of well-established nucleophilic substitution reactions.

\subsubsection{Reactions of $\mathbf{M}^{1}-\mathrm{M}^{2}$ Nucleophiles with Fluoro- arenes}

In 2016, we reported the reaction of fluoroarenes with 3, a compound containing a Mg-Mg bond (Figure 6a,b). ${ }^{46}$ Addition of the $\mathrm{C}-\mathrm{F}$ bond of a series of perfluorinated and polyfluorinated arenes across the $\mathrm{Mg}-\mathrm{Mg}$ bond of 3 proceeded rapidly in solution at $25{ }^{\circ} \mathrm{C}$. The reaction resulted in the formation of a new $\mathrm{Mg}-\mathrm{C}$ bond and a new $\mathrm{Mg}-\mathrm{F}$ bond and is analogous to Grignard formation in homogeneous solution. The products could be separated by fractional crystallisation following addition of an aliquot of THF, and were isolated as the etherate solvates. The magnesium organometallics demonstrated the expected four-coordinate geometry in the solid state as evidenced by single-crystal Xray diffraction studies. In solution, variable-temperature ${ }^{19} \mathrm{~F}$ NMR spectroscopy provided evidence for a weak residual interaction between the ortho-fluorine group of the aryl moiety and magnesium. Hence, at low temperature hin- 
dered rotation about the $\mathrm{Mg}-\mathrm{C}$ was implied by the observation of five distinct ${ }^{19} \mathrm{~F}$ NMR resonances for the pentafluorophenylmagnesium complex. At higher temperature two sets $\left(\mathrm{F}_{\text {ortho }}+\mathrm{F}_{\text {meta }}\right)$ of these resonances coalesce and the activation parameters of $\Delta \mathrm{H}^{\ddagger}=+7.6 \mathrm{kcal} \mathrm{mol}^{-1}$ and $\Delta \mathrm{S}^{\ddagger}=-15.1$ cal $\mathrm{K}^{-1} \mathrm{~mol}^{-1}$ provide an estimation of the maximum strength of the electrostatic $\mathrm{Mg} \ldots \mathrm{F}_{\text {ortho }}$ interaction, $\Delta \mathrm{G}^{\ddagger}$ $(298 \mathrm{~K})=+12.1 \mathrm{kcal} \mathrm{mol}^{-1} \cdot{ }^{46}$

The reaction scope includes 11 perfluorinated or partially fluorinated arenes. With 3 the scope is limited to activated fluorocarbons with at least four fluorine atoms, while the more reactive species $\mathbf{4}$ shows improved reactivity. In this case the complex nature of the reaction products prevents clean isolation and unambiguous confirmation of the products. More recently, Harder and co-workers isolated 5, a complex containing an extremely stretched $\mathrm{Mg}-\mathrm{Mg}$ bond, and showed that it reacts with fluorobenzene albeit under forcing conditions of $100{ }^{\circ} \mathrm{C}$ for five days (Figure 6a,b). ${ }^{47}$ The regioselectivity of $\mathrm{C}-\mathrm{F}$ activation is consistent with a nucleophilic substitution mechanism. Substitution of the arene with electron-withdrawing groups $\left(\mathrm{CF}_{3}, \mathrm{~N}, \mathrm{C}_{6} \mathrm{~F}_{5}, \mathrm{C}_{6} \mathrm{H}_{5}\right)$ leads to $\mathrm{C}-\mathrm{F}$ bond activation at the 4-position, while inclusion of an electron-donating group $\left(\mathrm{NMe}_{2}\right)$ begins to disfavour sub- stitution at this position, enriching the product from 3-substitution. Inclusion of a pyridyl directing group forces substitution at the adjacent site, the 2-position, through coordination of $\mathbf{3}$ to the heteroaromatic. In the case of partially fluorinated arenes, the regioselectivity is determined by the number and position of the unreactive $\mathrm{C}-\mathrm{F}$ bonds, with the site of substitution exclusively being flanked by one or two ortho $\mathrm{C}-\mathrm{F}$ bonds. This is a strict requirement for reactivity in the case of $\mathbf{3}$ and suggests that the ortho fluorine atom may be playing a role as a directing group.

In 2018, we expanded the study to include both experimental and computational appraisals of the reaction mechanism with further experiments to probe the nature of the proposed key transition state for $\mathrm{C}-\mathrm{F}$ bond activation. ${ }^{48} \mathrm{An}$ Eyring analysis was conducted on the reaction of $\mathbf{3}$ with $\mathrm{C}_{6} \mathrm{~F}_{6}$ across a $258-288 \mathrm{~K}$ temperature range allowing the activation parameters $\Delta \mathrm{H}^{\ddagger}=+10.8 \mathrm{kcal} \mathrm{mol}^{-1}$ and $\Delta \mathrm{S}^{\ddagger}=$ $-35 \mathrm{cal} \mathrm{K}^{-1} \mathrm{~mol}^{-1}$ to be determined. The large negative entropy of activation is consistent with a highly ordered transition state in the rate-determining step. The associated Gibbs activation energy is $\Delta \mathrm{G}^{\ddagger}(298 \mathrm{~K})=21.3 \mathrm{kcal} \mathrm{mol}^{-1}$. DFT calculations support a concerted pathway involving initial formation of a weak, unstable encounter complex followed (a)

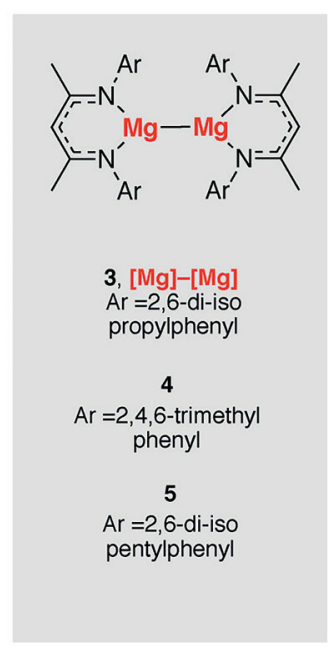

(c)

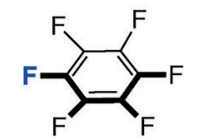

[Mg]-[Mg]

oxidative addition ( $C S_{N} A r$-like mechanism) (b)

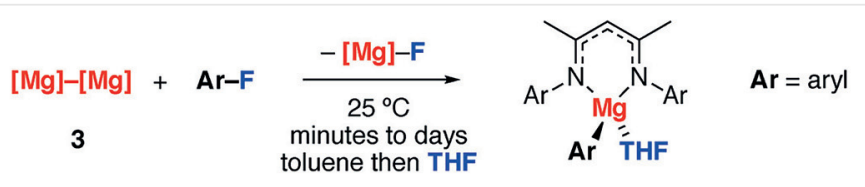

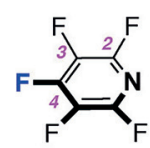

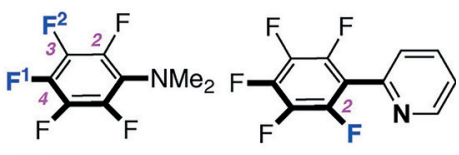

$F^{1}: F^{2}=3.4: 1$
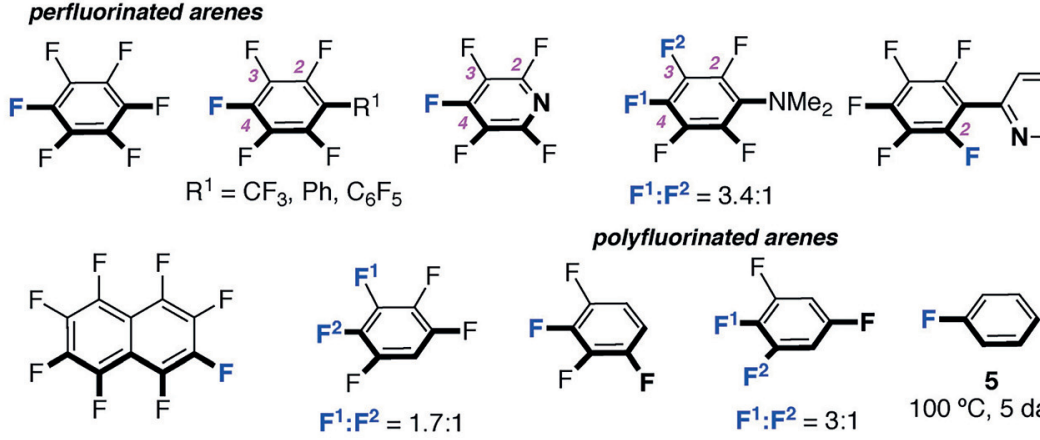

$F^{1}: F^{2}=3: 1$

$100^{\circ} \mathrm{C}, 5$ days
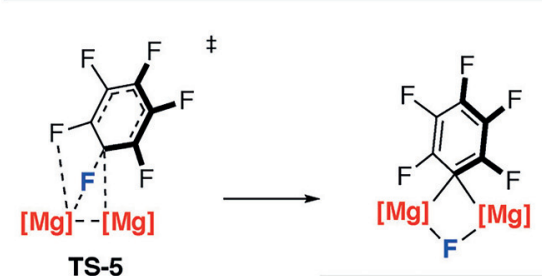

$\Delta \mathrm{H}^{\ddagger}=+10.8 \mathrm{kcal} \mathrm{mol}^{-1}$ $\Delta \mathrm{S}^{\ddagger}=-35 \mathrm{cal} \mathrm{K}^{-1} \mathrm{~mol}^{-1}$

$\Delta \mathrm{G}^{\ddagger}=+25.7 \mathrm{kcal} \mathrm{mol}^{-1}(\omega \mathrm{B} 97 \mathrm{x}-\mathrm{D})$
$\Delta \mathrm{G}^{\ddagger}=+22.7 \mathrm{kcal} \mathrm{mol}^{-1}(\mathrm{MO6L})$ $\Delta \mathrm{G}^{\ddagger}=+19.5 \mathrm{kcal} \mathrm{mol}^{-1}$ (B3PW91-D3)

Eyring Analysis

(d)

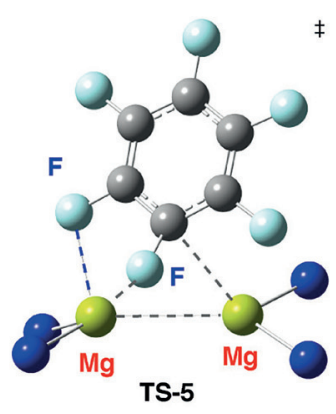

Figure 6 (a) Line-drawings of 3-5. (b) Reactions of Mg-Mg nucleophiles with fluoroarenes. (c) Proposed reaction mechanism based on DFT studies with experimentally determined activation parameters for the reaction of $\mathrm{C}_{6} \mathrm{~F}_{6}$ with 3. (d) Calculated geometry of TS-5. 
by $\mathrm{C}-\mathrm{F}$ bond cleavage by an $\mathrm{S}_{\mathrm{N}} \mathrm{Ar}$ pathway involving TS-5 (Figure 6c,d). Using different DFT functionals the calculated energy for TS-5 is in good agreement with the experimentally determined activation parameters, $\Delta \mathrm{G}^{\ddagger}(298 \mathrm{~K})=25.7$

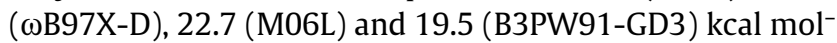
${ }^{1.48}$ In this transition state, one magnesium centre adopts the role of an electrophilic site to accept the fluoride leaving group, while the other acts as a nucleophile attacking the aromatic system and forming the new $\mathrm{Mg}-\mathrm{C}$ bond. The reaction bears all the hallmarks of a concerted nucleophilic aromatic substitution.

Deeper analysis of the transition state shows that electron density flows from the $\mathrm{Mg}-\mathrm{Mg}$ bond to the newly forming $\mathrm{Mg}-\mathrm{C}$ bond and from the $\mathrm{C}-\mathrm{F}$ bond to the fluoride leaving group. NBO calculations allow the interrogation of the charges as the reaction proceeds and these show that while both $\mathrm{Mg}$ atoms of $\mathbf{3}$ become more positive in TS-5, that which is accepting the fluorine atom bears the largest partial positive charge. TS-5 adopts a partial negative charge on the aromatic ring which is localised on the ipsocarbon and to a smaller extent the ortho and para carbon atoms.

One unexpected aspect of these calculations was the identification of short M...F interactions $(\sim 2.3 \AA)$ between ortho fluorine atoms and magnesium centres in TS-5. These interactions account for $\sim 5 \mathrm{kcal} \mathrm{mol}^{-1}$ stabilisation of the transition state and, along with the known trends for fluorine substitution on $\mathrm{C}-\mathrm{F}$ and $\mathrm{M}-\mathrm{F}$ bond strengths in fluoroarenes (see section 1.3), explain the observed experimental regioselectivity. $\mathrm{S}_{\mathrm{N}} A r$-type pathways have also been proposed for the reaction of related main group nucleophiles including $\left[\mathrm{LiB}(\mathrm{CN})_{2}\right]$ and $\left[\mathrm{LiSiR}_{3}\right]$ with fluoroarenes. ${ }^{49,50}$

Based on polarisation of the $\mathrm{Mg}-\mathrm{Mg}$ bond in the transition state for $\mathrm{C}-\mathrm{F}$ activation, we hypothesised that main group reagents containing polar $\mathrm{M}^{1}-\mathrm{M}^{2}$ bonds may be more reactive toward fluorocarbons. Our hope was that the ground state polarisation would improve rates of reactivity and ultimately a broader reaction scope. Using the same ligand system, a series of compounds containing $\mathrm{Mg}-\mathrm{Zn}$, $\mathrm{Mg}-\mathrm{Al}$ and $\mathrm{Zn}-\mathrm{Al}$ bonds were prepared (Figure 7a,b). All proved less reactive than the $\mathrm{Mg}-\mathrm{Mg}$ complex 3. Hence, only the $\mathrm{Mg}-\mathrm{Zn}$ compound $\mathbf{6}$ showed any reactivity toward fluorocarbons and this was limited to the activated substrates $\mathrm{C}_{6} \mathrm{~F}_{6}$ and 2-(pentafluorophenyl)pyridine. These reactions proceeded exclusively to form zinc organometallics and a magnesium fluoride by-product. Consistent with the polarisation arguments and the electronegativity difference of $\mathrm{Mg}$ and $\mathrm{Zn}$, there was no evidence for $\mathrm{Mg}-\mathrm{C}$ bond formation. Competition experiments between $\mathbf{3}$ and $\mathbf{6}$ showed that the $\mathrm{Mg}-\mathrm{Mg}$ nucleophile reacted at faster rates than the $\mathrm{Mg}-\mathrm{Zn}$ nucleophile. ${ }^{48}$

Ultimately calculations provided insight into the lack of reactivity of these polar $\mathrm{M}^{1}-\mathrm{M}^{2}$ bonds toward fluorocarbons. In all cases, concerted $\mathrm{S}_{\mathrm{N}} \mathrm{Ar}$ pathways could be calculated by DFT methods. These calculations showed that the energy of the transition state for $\mathrm{C}-\mathrm{F}$ bond activation of $\mathrm{C}_{6} \mathrm{~F}_{6}$ with main group nucleophiles TS-5 did not decrease with the polarisation of the metal-metal bond but rather followed the trend $\mathrm{Mg}-\mathrm{Mg}<\mathrm{Mg}-\mathrm{Zn}<\mathrm{Mg}-\mathrm{Al} \sim \mathrm{Al}-\mathrm{Zn}$ (Figure $7 \mathrm{a}, \mathrm{b})$. The simplest explanation for these results is that the steric and not electronic factors are dominating reactivity. Increasing the polarity difference between $\mathrm{M}^{1}$ and $\mathrm{M}^{2}$ results in an increased ionic character to the bond. In addition, both $\mathrm{Zn}(1.18 \AA)$ and $\mathrm{Al}(1.26 \AA)$ have smaller single bond covalent radii than $\mathrm{Mg}(1.39 \AA) .{ }^{51}$ Both effects manifest in a contraction of the metal-metal distance. Solventfree $\mathrm{Mg}-\mathrm{Zn}$ bonds are shorter than the $\mathrm{Mg}-\mathrm{Mg}$ bond length of 3 by $\sim 0.2 \AA$. This contraction brings the large and bulky ligands closer together and hinders access to the reactive site. Consistent with this argument, modification of the 2,6-

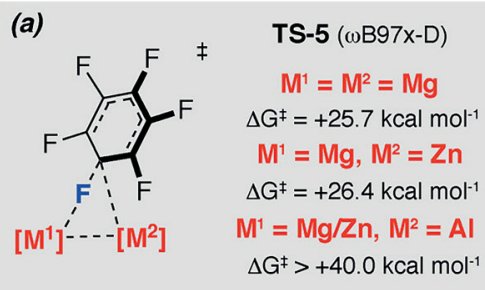

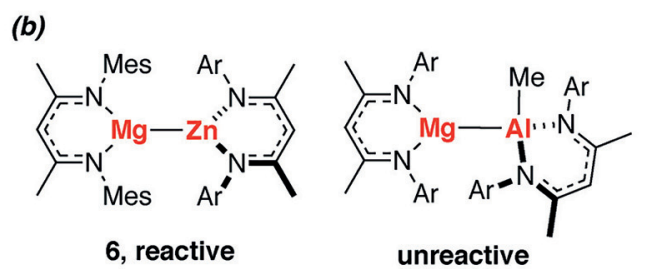

6 , reactive unreactive

$\mathrm{Ar}=2,6$-di-iso propylphenyl

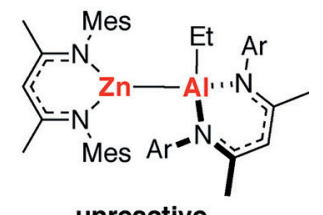

unreactive

(c)

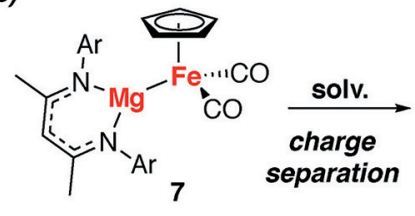

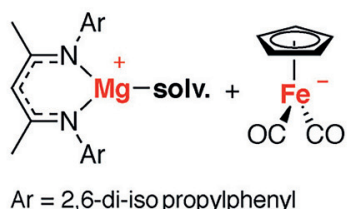

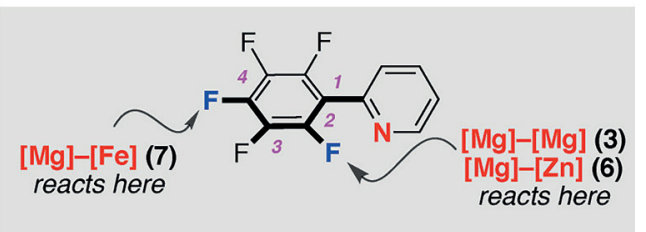

Figure 7 (a) Calculated Gibbs activation energies for TS-5 for a variety of $M^{1}-M^{2}$ nucleophiles. (b) line-drawings of $M^{1}-M^{2}$ nucleophiles prepared and tested in reactions with $\mathrm{C}_{6} \mathrm{~F}_{6}$ and related fluoroarenes. (c) Charge separation of $\mathrm{Mg}-\mathrm{Fe}$ complex $\mathbf{7}$ and (inset) regioselectivity in the reaction with 2(pentafluorophenyl)pyridine. 
diisopropylphenyl groups on $\mathbf{3}$ to the smaller mesityl groups on $\mathbf{4}$ results in improved rates of reaction, as evidenced by competition experiments, and a lower calculated TS energy for C-F activation. The hypothesis is further supported by the reactivity of $\mathbf{5}$ which, despite the bulky isopentyl groups on the ligand, shows a larger $\mathrm{Mg}-\mathrm{Mg}$ separation and improved reactivity with fluoroarenes. $\mathrm{Mg}-\mathrm{Mg}$ distances in $\mathbf{3}$ and $\mathbf{4}$ range from 2.808(1) to 2.846(1) $\AA$ while that in 5 is 3.056(1) $\AA .{ }^{47}$ For the $\mathrm{Mg}-\mathrm{Al}$ and $\mathrm{Zn}-\mathrm{Al}$ complexes, addition of an alkyl ligand on $\mathrm{Al}$ further serves to block access to the metal-metal bond and prevent nucleophilic attack on fluorocarbons. ${ }^{48}$

Further modification of the nucleophile to include an extremely polar $\mathrm{M}^{1}-\mathrm{M}^{2}$ bond leads to changes in the regioselectivity of addition and an apparent switch from a concerted nucleophilic substitution to a stepwise mechanism involving a genuine Meisenheimer intermediate. Hence, in 2019 we reported the reaction of a series of fluorocarbons with 7, a nucleophilic reagent containing a $\mathrm{Mg}-\mathrm{Fe}$ bond (Figure 7c). ${ }^{52} \mathrm{NBO}$ calculations and charge analysis, comparing 3 and 7, showed that while the former bond can be considered covalent apolar, the latter bond is almost entirely polarised toward $\mathrm{Mg}^{+}$and $\mathrm{Fe}^{-}$and primed for charge separation. In the presence of a substrate bearing a coordinating group, or indeed in highly polar coordinating solvents, 7 can undergo charge separation forming electrophilic and nucleophilic components that can engage in $\mathrm{C}-\mathrm{F}$ bond activation. DFT calculations again suggest a role for the Mg cation in acting to polarise the C-F bond toward nucleophilic attack and accept the fluoride anion. The differences between the reactivity of $\mathbf{3}$ and $\mathbf{7}$ are manifest in the selectivity for 2-(pentafluorophenyl)pyridine, with 3 reacting at the 2-position and $\mathbf{7}$ at the 4-position (Figure 7c). The calculations predict these selectivities for the concerted and stepwise pathways respectively. Furthermore, $\mathbf{7}$ appears to demonstrate improved conversions and reaction scope in polar solvent mixtures including THF or pyridine. ${ }^{52}$ The reactivity of 7 parallels that established back in the 1960s and 1970s for $\mathrm{NaFp}\left[\mathrm{Fp}=\mathrm{n}^{5}-\mathrm{C}_{5} \mathrm{H}_{5} \mathrm{Fe}(\mathrm{CO})_{2}\right]$ and related reagents by Stone and Bruce. ${ }^{53}$ More recently, related transition-metal complexes based on anionic Rh and Ir nucleophiles have been proposed to react with fluoroarenes..$^{54,55}$

\subsubsection{Reactions of $\mathbf{M}^{1}-\mathrm{M}^{2}$ Nucleophiles with Fluoroal- kanes}

Compound 3 also reacts with fluoroalkanes (Figure 8a). Linear, branched, cyclic and acyclic $1^{\circ}$ and $2^{\circ}$ fluoroalkanes react with 3 by 1,2-addition across the metal-metal bond under mild conditions $\left(50-80{ }^{\circ} \mathrm{C}\right.$; Figure $\left.8 \mathrm{~b}\right) .{ }^{56}$ The resulting alkyl magnesium products can dimerise in solution by three-centre, two-electron bonds and this is predicted to be favourable for products derived from $1^{\circ}$ fluoroalkanes. The reaction is stereoconvergent with both trans- and cis-4- tert-butyl-1-fluorocyclohexane reacting with $\mathbf{3}$ to form the trans-isomer of the resulting organometallic. While further experiments are necessary to probe the stereospecifity of the $\mathrm{Mg}-\mathrm{C}$ bond-forming step, it is likely that this stereoconvergence results from the epimerisation of the stereocentre adjacent to $\mathrm{Mg}$ in the product. DFT calculations suggest that the trans isomer of the product is more stable than the cis. Upon modification of the main group nucleophile to $\mathbf{4}$, the reaction scope can be expanded to $3^{\circ}$ alkyl fluorides, specifically 1-fluoroadamantane. In this instance, the resulting organomagnesium product is unstable with respect to Schlenk-like ligand redistribution, leading to the formation of $\mathrm{Ad}_{2} \mathrm{Mg}$ in situ ( $\mathrm{Ad}=1$-adamantyl). While the Schlenk equilibrium of the reaction products complicates their clean isolation, it does not prevent their efficient use in further synthetic steps.

We showed that the organomagnesium reagents derived from $\mathrm{C}-\mathrm{F}$ bond activation of 1 -fluorohexane can be used to transfer the hexyl group to a diverse range of electrophiles, proving $\mathrm{C}-\mathrm{Si}, \mathrm{C}-\mathrm{B}, \mathrm{C}-\mathrm{Sn}$ and even $\mathrm{C}-\mathrm{C}$ bond formation using a fragment derived from a fluorocarbon. This reactivity was capitalised on in order to develop the first transition-metal-free method to couple $\mathrm{sp}^{2} \mathrm{C}-\mathrm{F}$ and $\mathrm{sp}^{3} \mathrm{C}-\mathrm{F}$ bonds by initial reaction of $\mathbf{3}$ with 1-fluorohexane followed by addition of a perfluoroarene. The reactivity holds promise as a general means to upgrade fluorocarbons through cross-coupling. ${ }^{56}$ More recently, others have demonstrated that related main group nucleophiles, generated in situ from the reaction of $\mathrm{R}_{3} \mathrm{Si}$-Bpin with KOt-Bu or LiHMDS, will react with a range of fluoroalkanes $(B$ pin $=$ pinacolato borane; HMDS = hexamethyldisilazane) and effect a defluorosilylation. ${ }^{57,58}$

DFT calculations were used to probe the plausible mechanisms of $\mathrm{C}-\mathrm{F}$ bond activation. While we are yet to conduct a full mechanistic analysis, confidence in the accuracy of the calculations can be gained from prior studies of 3 with fluoroarenes (see section 2.2.1). Calculations suggest that for $1^{\circ}$ alkyl substrates, a remarkable $\mathrm{S}_{\mathrm{N}} 2$ pathway involving frontside nucleophilic attack of $\mathbf{3}$ on the fluorocarbon is energetically accessible (Figure $8 \mathrm{c}) .^{56}$ This pathway is a concerted process in which the polarisation of the substrate occurs through coordination of the $\mathrm{C}-\mathrm{F}$ bond to $\mathrm{Mg}$. The unusual frontside geometry of the transition state TS-6 can be rationalised on the basis of the nature of the reagents. The activation energy $\Delta \mathrm{G}^{\ddagger}(298 \mathrm{~K})=23.6 \mathrm{kcal} \mathrm{mol}^{-1}$ of TS-6 was determined using the B3PW91-GD3 functional. Complex 3 contains a covalent apolar metal-metal bond and has been proposed to possess a non-nuclear attractor in the interatomic region: due to the highly electropositive magnesium atoms, electron density is localised in the centre of the two Mg nuclei. ${ }^{59,60}$

In the transition state for frontside nucleophilic attack, the electropositive $\mathrm{Mg}$ sites coordinate the electronegative fluorine atom of the fluorocarbon, orientating it front-on. 

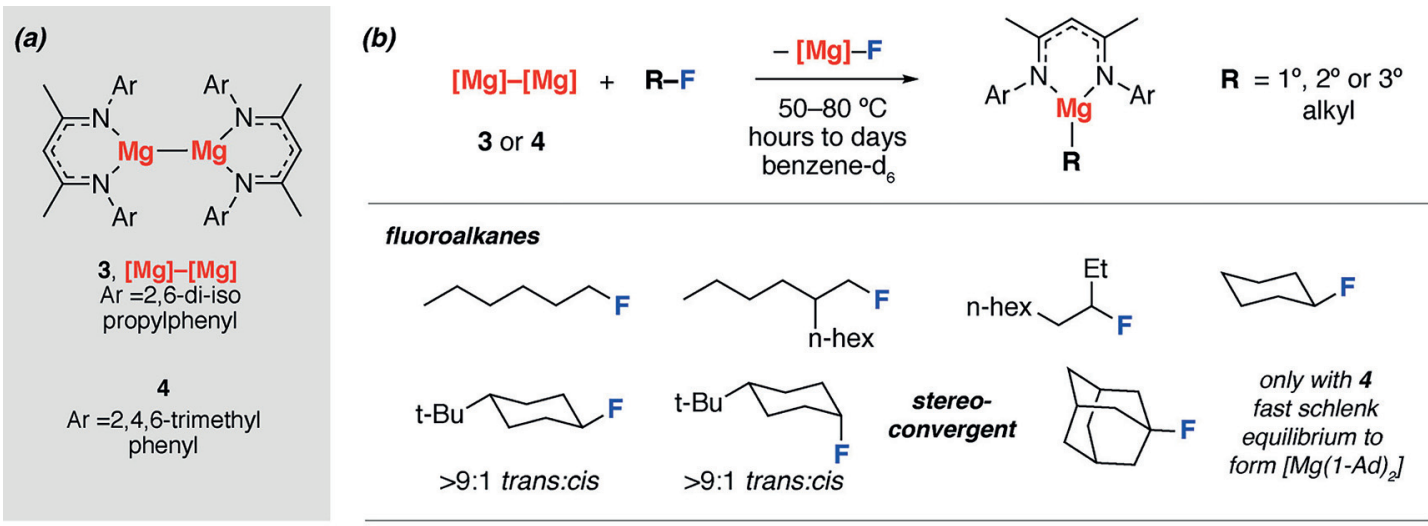

(c)
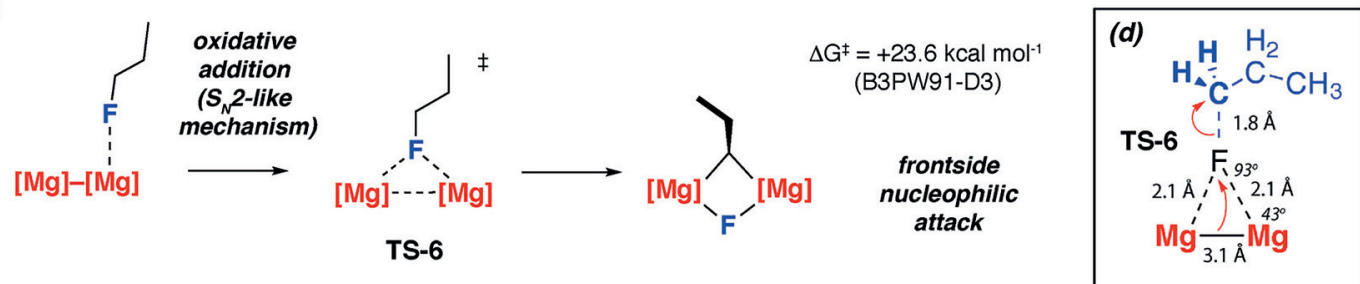

Figure 8 (a) Line-drawings of 3 and 4. (b) Reactions of Mg-Mg nucleophiles with fluoroalkanes. (c) Proposed reaction mechanism based on DFT studies for reaction of $n$-PrF with 3. (d) Calculated geometry of TS-6 showing frontside nucleophilic attack.

Coordination acts to polarise the $\mathrm{C}-\mathrm{F}$ bond and nucleophilic attack can occur with flow of electron density from the $\mathrm{Mg}-\mathrm{Mg} \sigma$-bond to the $\sigma^{*}$ orbital of the $\mathrm{C}-\mathrm{F}$ bond. In this reaction the carbon centre is the leaving group and the reactive carbon centre generates carbanion character as it migrates to the Mg centres in the product.

\subsubsection{Reactions of $M^{1}-M^{2}$ Nucleophiles with Fluoro- alkenes}

Attempts to develop similar reactivity of $\mathbf{3}$ with fluoroalkenes led to undesired side-reactions. For example, HFP reacts with $\mathbf{3}$ to form a complex mixture that includes a major product derived from $\mathrm{C}-\mathrm{C}$ bond formation due to nucleophilic attack of the $\beta$-diketiminate ligand on the fluorocarbon. The observation that ligand reactivity competes with metal-based reactivity can be explained by considering a localised resonance form of the ligand and its role as a metal enamide. The undesired pathway could be avoided by a combination of modification of the ligand system and variation of the metal-metal bond. Hence a number of magnesium and lithium silyl reagents were shown to be highly effective nucleophiles for the defluorosilylation of HFP and related industrially relevant fluoroalkenes (Figure 9a,b). ${ }^{61}$

Of the nucleophiles studied, lithium silyl reagents proved the most effective. HFP, HFO-1234yf, HFO-1234ze, HFO-1336mzz and 3,3,3-trifluoroprop-1-ene reacted with [Li(SiPhMe 2$\left.)(\mathrm{THF})_{1.5}\right]$ in benzene or THF solution at $25^{\circ} \mathrm{C}$ or below within 15 minutes to 3 hours to give a high yield of the defluorosilylated products (Figure 9a,b). In the case of
HFO-1234yf, a selective second addition of the silyl lithium reagent could be achieved leading to a doubly defluorosilylated product. Modification of the ligand on lithium to chelating amines such as TMEDA or PMDETA led to lower yields of $\mathrm{C}-\mathrm{F}$ activation. While the analogous magnesium reagents $\mathbf{9}$ and $\mathbf{1 0}$ were also competent for defluorosilylation, they required higher reaction temperatures (60-100 ${ }^{\circ} \mathrm{C}$ ) and longer reaction times (3-6 days) to effect the same transformation as the lithium analogues. Consistent with the steric arguments observed in the reactions of $\mathbf{3}$ with fluoroarenes (see section 2.2.1), magnesium nucleophiles bearing the less sterically hindered ligands demonstrated improved reaction scope over those with bulky ligand systems.

Two reactivity patterns were observed with all the sblock nucleophiles investigated. Substrates containing terminal $\mathrm{sp}^{2} \mathrm{C}-\mathrm{F}$ bonds (HFP and HFO-1234ze) undergo a stereospecific $\mathrm{S}_{\mathrm{N}} \mathrm{V}$ reaction leading to direct silylation of these bonds with conservation of the stereochemistry of the $C=C$ double bond. In contrast, substrates that possess either internal or no $\mathrm{sp}^{2} \mathrm{C}-\mathrm{F}$ bonds underwent an $\mathrm{S}_{\mathrm{N}} 2^{2}$ pathway ultimately leading to cleavage of an $\mathrm{sp}^{3} \mathrm{C}-\mathrm{F}$ bond of the vinyl $\mathrm{CF}_{3}$ group. This latter pathway is especially notable for HFO1234 yf as reaction with the aluminium(I) reagent 1 leads exclusively to substitution at the internal $\mathrm{sp}^{2} \mathrm{C}-\mathrm{F}$ bond (see section 2.1.2).

DFT calculations support the proposed mechanisms and provide qualitative explanations for the observed reactivity trends (Figure 9c). These calculations focused on the magnesium silyl reagents $\mathbf{9}$ and $\mathbf{1 0}$ due to their defined coordi- 


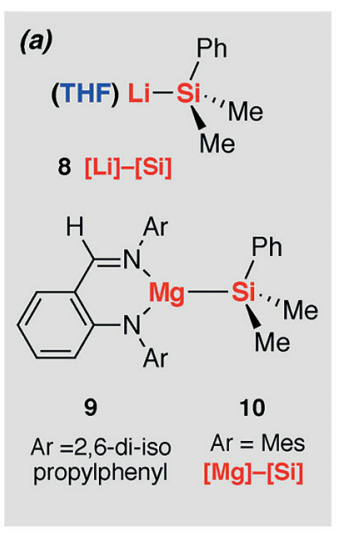

(c) $S_{N} V$ pathway

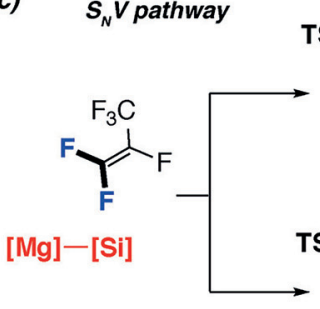

(b)

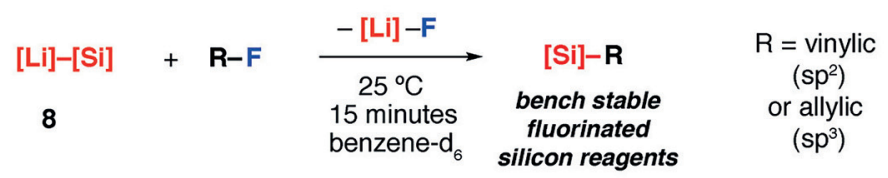

$S_{N} V$ pathway<smiles>F/C=C/C#[As]</smiles><smiles>FC(F)=C(F)F</smiles><smiles>C=C[AsH3-]</smiles><smiles>[C+]#CC=CC</smiles><smiles>C=C(F)F</smiles>

HFO-1234ze HFP stereospecific $F^{1}: F^{2}=>9: 1$

HFO-1336mzz HFO-1234yf

$S_{N} 2$ ' pathway chemoselective for allylic $C-F$ nation geometry and known ligand sphere. Low-energy pathways were again found to be those that were concerted and involved the main group reagent taking a dual role of nucleophile attacking a carbon centre and electrophilic site to accept the fluoride leaving group and polarise the $\mathrm{C}-\mathrm{F}$ bond.

Due to the electronegativity difference of $\mathrm{Mg}$ and $\mathrm{Si}$ and the polarisation of the $\mathrm{Mg}-\mathrm{Si}$ bond, the $\mathrm{Mg}$ site accepts the F leaving group while the Si site acts as a nucleophile. For HFP a number of low-energy transition states for a concerted $\mathrm{S}_{\mathrm{N}} \mathrm{V}$ mechanism could be found. Hence, with the B3PW91-GD3 functional, TS-7 $\Delta \mathrm{G}^{\ddagger}(298 \mathrm{~K})=14.7<\mathrm{TS}-8 \Delta \mathrm{G}^{\ddagger}$ $(298 \mathrm{~K})=16.6 \mathrm{kcal} \mathrm{mol}^{-1}$. While TS-7 and TS-8 give rise to the experimentally observed products from attack of the nucleophile at the terminal $\mathrm{sp}^{2} \mathrm{C}-\mathrm{F}$ bonds, further transition states that arise from $S_{N} V$ of the internal $\mathrm{sp}^{2} \mathrm{C}-\mathrm{F}$ position or an $\mathrm{S}_{\mathrm{N}} 2^{\prime}$ pathway are less energetically accessible, and the corresponding products are not observed experimentally. For HFO-1234yf, a concerted $\mathrm{S}_{\mathrm{N}} 2$ ' mechanism was calculated to proceed by TS-9 with $\Delta \mathrm{G}^{\ddagger}(298 \mathrm{~K})=22.5 \mathrm{kcal} \mathrm{mol}^{-1}$. TS-9 involves allylic $\mathrm{sp}^{3} \mathrm{C}-\mathrm{F}$ bond activation. Dual activation of the substrate occurs, but now that site of nucleophilic attack is two bonds removed from the C-F bond that breaks. ${ }^{61}$

\section{Summary and Perspective}

In summary, over the last eight years we have developed a number of novel reactions that involve the addition of main group nucleophiles to fluorocarbons. These reactions either involve the 1,1-addition of the R-F bond to a singlesite metal complex or 1,2-addition of the R-F bond across an $\mathrm{M}-\mathrm{M}$ or $\mathrm{M}-\mathrm{Si}$ bond. The new reactivity builds on the findings of others and exploits main group molecules pioneered by a number of groups over the past two decades. Through a combination of physical organic experiments (competition experiments, rate-laws, activation parameters) and calculations (DFT, QTAIM) we have attempted to develop a deep mechanistic understanding of the new reactivity. While many subtleties are yet to be understood, the global picture that emerges is that strong electrostatic interactions between electropositive main group elements and fluorine atoms play key roles in determining both the thermodynamics and the kinetics of the reaction pathways.

A number of challenges still remain in this field. From the perspective of recycling and upgrading fluorinated gases, HFCs remain the least reactive substrates. For example, new reactions to selectively convert the $\mathrm{sp}^{3} \mathrm{C}-\mathrm{F}$ bonds of tri- 
fluoromethane or 1,1,1,2-tetrafluoroethane into $\mathrm{sp}^{3} \mathrm{C}$-Si or $\mathrm{sp}^{3} \mathrm{C}-\mathrm{B}$ bonds would be of significant value to the synthetic community due to the abundance of these fluorinated gases. ${ }^{62}$ This goal will require new selective reactions of a single $\mathrm{sp}^{3} \mathrm{C}-\mathrm{F}$ bond of the $\mathrm{CF}_{3}$ group to be developed. From the perspective of late-stage functionalisation of complex molecules, there is a need to try and understand the scope and functional group tolerance of the new reactivity. Functional-group-tolerant methods could well find application in the late-stage modification of fluorine containing drugs and provide new methods to derivatise and label complex organic molecules. We look forward to learning more about the existing systems and embracing these challenges in the coming years.

\section{Funding Information}

We are grateful to the European Research Council for support in the form of an ERCstG (Fluorofix: 677367) and Marie Curie Sponsporship (Fluorocat and Fluorocross). GC is thankful for EPSRC funding in the form of a DTP scholarship.

\section{Acknowledgment}

M.R.C. gratefully acknowledges the highly talented co-workers who have contributed to this project over the last eight years. Without them none of the work would have been possible.

\section{References}

(1) Fluorinated Polymers: Applications, Vol. 2; Ameduri, B.; Sawada, H., Ed.; Royal Society of Chemistry: Cambridge, 2016, 1-372.

(2) O'Hagan, D. J. Fluorine Chem. 2010, 131, 1071.

(3) Harsanyi, A.; Sandford, G. Green Chem. 2015, 17, 2081.

(4) Fowler, R. D.; Burford, W. B. III.; Hamilton, J. M. Jr.; Sweet, R. G.; Weber, C. E.; Kasper, J. S.; Litant, I. Ind. Eng. Chem. 1947, 39, 292.

(5) Simons, J. H. J. Electrochem. Soc. 1949, 95, 47.

(6) O'Hagan, D. Chem. Soc. Rev. 2008, 37, 308.

(7) Blanksby, S. J.; Ellison, G. B. Acc. Chem. Res. 2003, 36, 255.

(8) Evans, M. E.; Burke, C. L.; Yaibuathes, S.; Clot, E.; Eisenstein, O.; Jones, W. D. J. Am. Chem. Soc. 2009, 131, 13464.

(9) Clot, E.; Mégret, C.; Eisenstein, O.; Perutz, R. N. J. Am. Chem. Soc. 2009, 131, 7817.

(10) Clot, E.; Eisenstein, O.; Jasim, N.; Macgregor, S. A.; McGrady, J. E.; Perutz, R. N. Acc. Chem. Res. 2011, 44, 333.

(11) Macgregor, S. A.; McKay, D.; Panetier, J. A.; Whittlesey, M. K. Dalton Trans. 2013, 42, 7386.

(12) Ricci, P.; Krämer, K.; Cambeiro, X. C.; Larrosa, I. J. Am. Chem. Soc. 2013, 135, 13258.

(13) Weaver, J.; Senaweera, S. Tetrahedron 2014, 70, 7413.

(14) Rohrbach, S.; Smith, A. J.; Pang, J. H.; Poole, D. L.; Tuttle, T.; Chiba, S.; Murphy, J. A. Angew. Chem. Int. Ed. 2019, 49, 569.

(15) Kwan, E. E.; Zeng, Y.; Besser, H. A.; Jacobsen, E. N. Nat. Chem. 2018, 10, 917.

(16) Aizenberg, M.; Milstein, D. Science 1994, 265, 359.

(17) Whittlesey, M. K.; Perutz, R. N.; Moore, M. H. Chem. Commun. 1996, 787.
(18) Pike, S. D.; Crimmin, M. R.; Chaplin, A. B. Chem. Commun. 2017, $53,3615$.

(19) Chen, W.; Bakewell, C.; Crimmin, M. Synthesis 2017, 49, 810.

(20) Eisenstein, O.; Milani, J.; Perutz, R. N. Chem. Rev. 2017, 117, 8710.

(21) Kiplinger, J. L.; Richmond, T. G.; Osterberg, C. E. Chem. Rev. 1994, 94, 373.

(22) Braun, T.; Wehmeier, F. Eur. J. Inorg. Chem. 2011, 613.

(23) Jones, W. D. Dalton Trans. 2003, 3991.

(24) Lentz, D.; Braun, T.; Kuehnel, M. F. Angew. Chem. Int. Ed. 2013, $52,3328$.

(25) Hughes, R. P. Eur. J. Inorg. Chem. 2009, 4591.

(26) Weetman, C.; Inoue, S. ChemCatChem 2018, 10, 4213.

(27) Cui, C.; Roesky, H. W.; Schmidt, H.-G.; Noltemeyer, M.; Hao, H.; Cimpoesu, F. Angew. Chem. 2000, 39, 4274.

(28) Crimmin, M. R.; Butler, M. J.; White, A. J. P. Chem. Commun. 2015, 51, 15994.

(29) Ekkert, O.; Strudley, S. D. A.; Rozenfeld, A.; White, A. J. P.; Crimmin, M. R. Organometallics 2014, 33, 7027.

(30) Yow, S.; Gates, S. J.; White, A. J. P.; Crimmin, M. R. Angew. Chem. Int. Ed. 2012, 51, 12559.

(31) Chen, W.; Hooper, T. N.; Ng, J.; White, A. J. P.; Crimmin, M. R. Angew. Chem. Int. Ed. 2017, 56, 12687.

(32) Chu, T.; Korobkov, I.; Nikonov, G. I. J. Am. Chem. Soc. 2014, 136, 9195.

(33) Samuel, P. P.; Singh, A. P.; Sarish, S. P.; Matussek, J.; Objartel, I.; Roesky, H. W.; Stalke, D. Inorg. Chem. 2013, 52, 1544.

(34) Jana, A.; Samuel, P. P.; Tavčar, G.; Roesky, H. W.; Schulzke, C. J. Am. Chem. Soc. 2010, 132, 10164.

(35) Chu, T.; Boyko, Y.; Korobkov, I.; Nikonov, G. I. Organometallics 2015, 34, 5363.

(36) Villegas-Escobar, N.; Gutiérrez-Oliva, S.; Toro-Labbé, A. J. Phys. Chem. C 2015, 119, 26598.

(37) Zhang, X.; Cao, Z. Dalton Trans. 2016, 45, 10355.

(38) García-Rodeja, Y.; Bickelhaupt, F. M.; Fernandez, I. Chem. Eur. J. 2016, 22, 13669.

(39) Jain, S.; Vanka, K. Chem. Eur. J. 2017, 23, 13957.

(40) Harder, S.; Brand, S.; Elsen, H.; Langer, J.; Donaubauer, W. A.; Hampel, F. Angew. Chem. Int. Ed. 2018, 57, 14169.

(41) Kim, Y.; Cho, H.; Hwang, S. Bull. Korean Chem. Soc. 2017, 38, 282.

(42) Pitsch, C. E.; Wang, X. Chem. Commun. 2017, 53, 8196.

(43) Choi, J.; Wang, D. Y.; Kundu, S.; Choliy, Y.; Emge, T. J.; Krogh-Jespersen, K.; Goldman, A. S. Science 2011, 332, 1545.

(44) Bakewell, C.; White, A. J. P.; Crimmin, M. R. Angew. Chem. Int. Ed. 2018, 57, 6638.

(45) Bakewell, C.; White, A. J. P.; Crimmin, M. R. Chem. Sci. 2019, 10, 2452.

(46) Bakewell, C.; White, A. J. P.; Crimmin, M. R. J. Am. Chem. Soc. 2016, 138, 12763.

(47) Gentner, T. X.; Rösch, B.; Ballmann, G.; Langer, J.; Elsen, H.; Harder, S. Angew. Chem. Int. Ed. 2019, 58, 607.

(48) Bakewell, C.; Ward, B. J.; White, A. J. P.; Crimmin, M. R. Chem. Sci. 2018, 9, 2348.

(49) Landmann, J.; Hennig, P. T.; Ignat'ev, N. V.; Finze, M. Chem. Sci. 2017, 8, 5962 .

(50) Mallick, S.; Xu, P.; Würthwein, E.-U.; Studer, A. Angew. Chem. Int. Ed. 2019, 58, 283.

(51) Pyykkö, P.; Atsumi, M. Chem. Eur. J. 2009, 15, 186.

(52) Garçon, M.; Bakewell, C.; White, A. J. P.; Crimmin, M. R. Chem. Commun. 2019, 55, 1805.

(53) Bruce, M. I.; Stone, F. G. A. Angew. Chem. Int. Ed. 1968, 7, 747.

(54) Peterson, T. H.; Golden, J. T.; Bergman, R. G. Organometallics 1999, 18, 2005. 
(55) Edelbach, B. L.; Jones, W. D. J. Am. Chem. Soc. 1997, 119, 7734.

(56) Crimmin, M. R.; Coates, G.; Bakewell, C.; Ward, B.; White, A. Chem. Eur. J. 2018, 24, 16282.

(57) Martin, R.; Liu, X.-W.; Zarate, C. Angew. Chem. Int. Ed. 2018, 58, 2064.

(58) Cui, B.; Jia, S.; Tokunaga, E.; Shibata, N. Nat. Commun. 2018, 9, 4393.
(59) Stasch, A.; Jones, C. Dalton Trans. 2011, 40, 5659.

(60) Overgaard, J.; Jones, C.; Stasch, A.; Iversen, B. B. J. Am. Chem. Soc. 2009, 131, 4208.

(61) Coates, G.; Tan, H. Y.; Kalff, C.; White, A. J. P.; Crimmin, M. R. Angew. Chem. Int. Ed. 2019, 58, 12514.

(62) Ito, S.; Kato, N.; Mikami, K. Chem. Commun. 2017, 53, 5546. 